\title{
EXAMPLES OF BIFURCATION FROM A CONTINUUM OF EIGENVALUES AND FROM THE CONTINUOUS SPECTRUM
}

By

\author{
M. C. JORGE AND A. A. MINZONI
}

Universidad Nacional Autonoma de Mexico

\begin{abstract}
In this note we consider an example arising from a caricature of a geophysical problem where we show explicitly bifurcation from a continuum of eigenvalues. Also we consider an example of bifurcation from the continuous spectrum for a simple integrodifferential equation.
\end{abstract}

Introduction. In the numerical study of bifurcation for an oblique derivative problem [2] we found bifurcation from the edge of a continuum of eigenvalues. Also we found isolated points in the continuum of eigenvalues where it was possible to perform the Lyapounov-Schmidt decomposition and show local bifurcation. The behavior at the other points of the continuum has not been determined. In this note we study a simple model problem which shows what may happen in the full problem. In particular, each point of the continuum of eigenvalues is a bifurcation point but the bifurcated branches are tangent to the trivial solution at each point of the continuum. Moreover, along each bifurcation branch there is a different rate of decay for the solution. This rate is used as a label for the branches.

We also show in a simple problem how tangency of bifurcated branches can occur when the solutions bifurcate from the continuous spectrum and are labelled by the frequency of the solutions. This behavior is very different from the one found in previous studies of other problems of bifurcation from the continuous spectrum.

Formulation. In [2] we study the problem of finding the electrostatic potential $\Psi$ in the exterior of a sphere given measurements $g(\theta, \varphi)$ of $|\nabla \Psi|$ on the surface. The problem to be solved is (in spherical coordinates)

$$
\begin{aligned}
\Delta \Psi & =0 \quad \text { for } r>1, \\
\nabla \Psi \cdot \nabla \Psi & =g(\theta, \varphi) \quad \text { on } r=1, \\
\Psi & \sim \frac{1}{r} \quad \text { as } r \rightarrow \infty .
\end{aligned}
$$

The special case considered in detail in [2] is for $g(\theta, \varphi)=\left|\nabla\left(r^{-1}+\mu r^{-2} \cos \theta\right)\right|$. 
Taking $\Psi=r^{-1}+\mu r^{-2} \cos \theta+v$ we obtain a bifurcation problem for $v$ in the form

$$
\begin{gathered}
\Delta v=0, \\
(2+4 \mu \cos \theta) \frac{\partial v}{\partial n}-2 \mu \sin \theta \frac{\partial v}{\partial \theta}+\nabla v \cdot \nabla v=0 \quad \text { on } r=1, \\
v \sim \frac{1}{r} \quad \text { as } r \rightarrow \infty .
\end{gathered}
$$

The problem can be transformed into a discrete problem expanding in Legendre polynomials (which produce solutions independent of the azimuth). Taking $\lambda=\mu^{-1}$,

$$
v(\lambda, r, \theta)=\sum_{n=0}^{\infty} r^{-(n+1)} v_{n}(\lambda) P_{n}(\cos \theta)
$$

we obtain, after substitution in the equations and the appropriate projections, that the amplitudes $v_{n}(\lambda)$ satisfy

$$
\frac{3(n+2)}{2 n+3} v_{n+1}(\lambda)+\frac{n}{2 n-1} v_{n-1}(\lambda)-\frac{1}{\mu} v_{n}(\lambda)=Q(\mathbf{v}(\lambda))
$$

where the quadratic nonlinearity $Q$ takes the form

$$
Q(\mathbf{v}(\lambda))=\sum_{0 \leq p, q \leq n} C_{n p q} v_{p}(\lambda) v_{q}(\lambda),
$$

where the coefficients $C_{n p q}$ are given. Notice that the sum truncates at $p=q=n$ due to the orthogonality of the Legendre polynomials. It was shown in [2] that the linearized equation has a continuum of eigenvalues for $|\mu| \geq \frac{1}{2}$. It was also shown that there exist some privileged points $\mu_{n}$ where the Lyapunov-Schmidt reduction can be performed to obtain local branches bifurcating from these points.

At the other points it is not possible. The nature of the bifurcations at the other points remains to be resolved.

To mimic the behavior of the system we retain the essential features, namely, the continuum of eigenvalues and the quadratic nature of the nonlinearity. Also quadratic nonlinearities produce convolution-like nonlinearities of their Legendre expansion. We thus take as the model equations the equations

$$
\lambda a_{n+1}-a_{n}=\sum_{m=0}^{n} a_{n-m} a_{m} \text { for } n \geq 0
$$

and search for solutions that satisfy $\sum_{n=0}^{\infty} a_{n}^{2}<\infty$.

Study of the model equations. The linearization for Eq. (1) has solutions $a_{n}=$ $a_{0} \lambda^{-n}$. For $|\lambda|>1$ the solutions are square summable and they are the continuum of eigenfunctions and $|\lambda|>1$ is the continuum of eigenvalues.

To study the nonlinear problem introduce the generating function

$$
G(z)=\sum_{n=0}^{\infty} a_{n} z^{n}, \quad a_{n}=\frac{1}{2 \pi i} \int_{C} \frac{G(z)}{z^{n+1}} d z .
$$

Clearly the decay of the $a_{n}$ is determined by the position of the singularities of $G(z)$. If $G(z)$ is regular up to $|z|=R>1$ then the decay of the $a_{n}$ is like $R^{-n}$. 
For Eq. (1) multiplication by $z^{n}$ and summation give the simple algebraic equation

$$
z G^{2}(z)+(z-\lambda) G(z)+\lambda a_{0}=0 .
$$

The solution has to be regular at $z=0$. This gives

$$
G(z)=\frac{(\lambda-z)-\sqrt{(\lambda-z)^{2}-4 z \lambda a_{0}}}{2 z} .
$$

The function $G(z)$ has a branch point at $(\lambda-z)^{2}-4 z \lambda a_{0}=0$. To obtain an acceptable solution we need $|z|>1$ for the branch point and $z$ real since we want real solutions.

If we fix $z=p$ real, $|p|>1$, along each curve

$$
a_{0}=\frac{(\lambda-p)^{2}}{4 p \lambda}
$$

the solution decays like $p^{-n}$. Each curve is tangent to the trivial solution at $\lambda=$ $p$. For this reason this branch cannot be captured via the usual Lyapunov-Schmidt decomposition since the nontrivial solution is not transverse to the trivial branch.

The bifurcation diagram shown in Fig. 1 is given by the family of curves of Eq. (2) parametrized by $p$. Along each curve the coefficients $a_{n}$ have different decays. For each value of $|\lambda|>1$ there are infinitely many solutions parametrized by the rate of decay $p$.

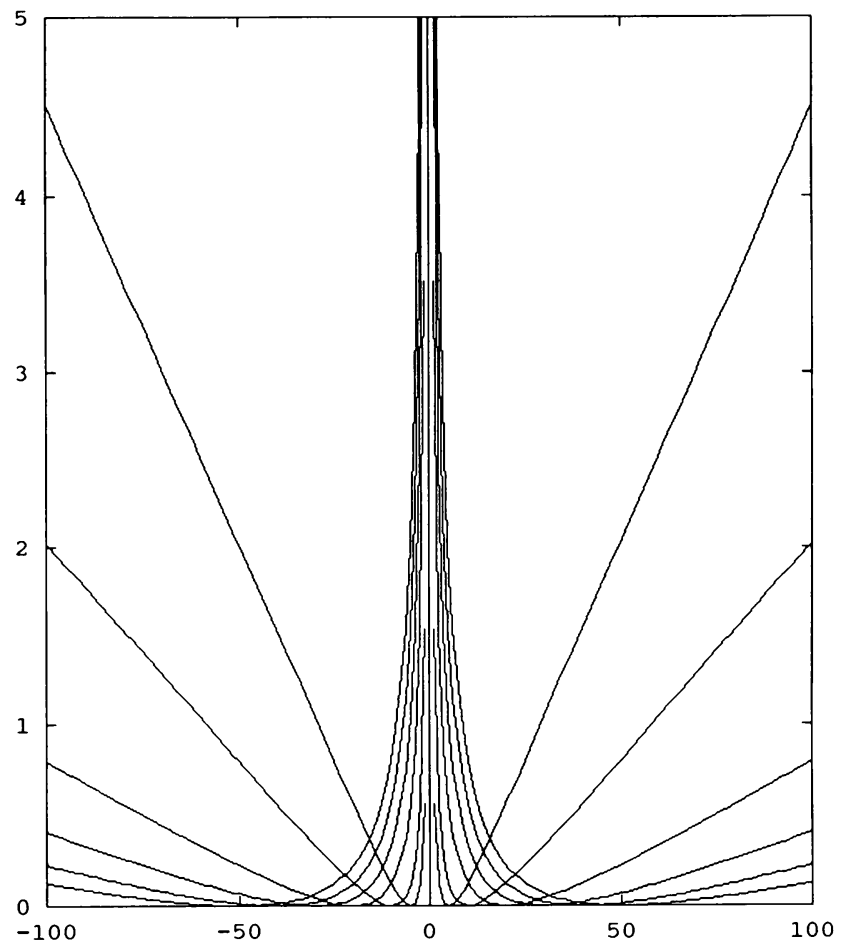

Fig. 1. Graph of $a_{0}=(\lambda-p)^{2} / 4 \lambda p$ for $p= \pm 5, \pm 10, \pm 20, \pm 30, \pm 40, \pm 50$. 
To our knowledge there are no examples available in the literature concerning bifurcation from a continuum of eigenvalues. Studies of bifurcation from the essential spectrum concern bifurcation from the continuous spectrum [1-5]. In the next section we describe these results and give a simple example that again exhibits a behavior different from the one found in previous studies.

A simple example of bifurcation from the continuous spectrum. The problems studied by Benci, Fortunato, Küpper, Stuart, and Toland [1-6] concern the existence of multiple solutions for equations of the form

$$
\begin{gathered}
-\Delta u+G(x)|u|^{p-2}=\lambda u, \quad p>2, \\
|u| \rightarrow 0 \text { as }|x| \rightarrow \infty,
\end{gathered}
$$

or appropriate one-dimensional versions [6] on the half line with a given boundary condition at $x=0$. In these studies it is shown that there are infinitely many branches for $\lambda \geq 0$ coalescing at $u=\lambda=0$ [6]. Moreover, Toland has shown [6] that these branches are the limit (as the length increases) of the global branches bifurcating from the eigenvalues of the Neumann problem for a finite interval. The work of Benci and Fortunato [1] shows a similar behavior at every point $\lambda \geq 0$ of the continuous spectrum of the linearized equation.

We now show in a simple example that at each point of the continuous spectrum there is a branch of solutions tangent to it. This behavior is different from the behavior observed in previous studies, which show coalescence of branches of a different number of nodes at each point of the spectrum $[3,4]$.

The example we consider is the nonlocal equation

$$
y^{\prime \prime}(x)+\lambda y(x)=\int_{0}^{x} y(x-\xi) y(\xi) d \xi \text { for } x \geq 0,
$$

$y(0)=0, y(x)$ bounded as $x \rightarrow \infty$.

The linear problem has a continuous spectrum $\lambda \geq 0$ with generalized eigenfunctions $y_{\lambda}(x)=\sin \sqrt{\lambda} x$. Again it is not possible to perform the Lyapunov-Schmidt reduction.

Denoting by $\tilde{y}(s)$ the transform of $y(x)$ we obtain

$$
s^{2} \tilde{y}(s)-y^{\prime}(0)+\lambda \tilde{y}=\tilde{y}^{2} .
$$

The solution for $\tilde{y}(s)$ that decays as $s \rightarrow 0, \operatorname{Re} s>0$ is given by

$$
\tilde{y}(s)=\left(s^{2}+\lambda\right)-\sqrt{\left(s^{2}+\lambda\right)^{2}-4 y^{\prime}(0)} .
$$

The solution $y(x)$ is given by

$$
y(x)=\frac{1}{2 \pi i} \int_{C} e^{s x}\left\{\left(s^{2}+\lambda\right)-\sqrt{\left(s^{2}+\lambda\right)^{2}-4 y^{\prime}(0)}\right\} d s .
$$


If the branch points are located in $\operatorname{Re} s \leq 0$ then the integral will lead to an acceptable solution. The position of the branch points is easily determined. They are at the values of $s$ that satisfy $\left(s^{2}+\lambda\right)^{2}=4 y^{\prime}(0)$. If $y^{\prime}(0)<0$ we obtain, solving for $s$,

$$
s^{2}=-\lambda \pm i 2 \sqrt{\left|y^{2}(0)\right|},
$$

which leads to branch points in $\operatorname{Re} s>0$ and, therefore, to growing solutions.

If $y^{\prime}(0) \geq 0$ the position of the branch points satisfies $\pm \sqrt{y^{\prime}(0)}=\lambda+s^{2}$. The only possibility for bounded real solutions is with branch points at $s= \pm i p, p$ real.

This gives $y^{\prime}(0)=\left(\lambda-p^{2}\right)^{2}$. Therefore the branches of solutions given by the family of parabolas $y^{\prime}(0)=\left(\lambda-p^{2}\right)^{2}$ bifurcate tangentially from the continuous spectrum $\lambda \geq 0$. The corresponding diagram is shown in Fig. 2 .

The branches are labeled by $p$. In this case $p$ does not label the rate of decay, which is $O\left(x^{-1}\right)$ for all the branches, but labels the frequency of oscillation. This is readily seen by changing $s=i p+\mu$ and using Watson's lemma on the integral representation.

Again we obtain for each $\lambda \geq 0$ a family of branches bifurcating tangentially from the trivial solution. Notice that the bifurcated solutions decay as $x \rightarrow \infty$, which is not the case for the solutions of the linearization. In this case the solution branches are labeled by this frequency of oscillation.

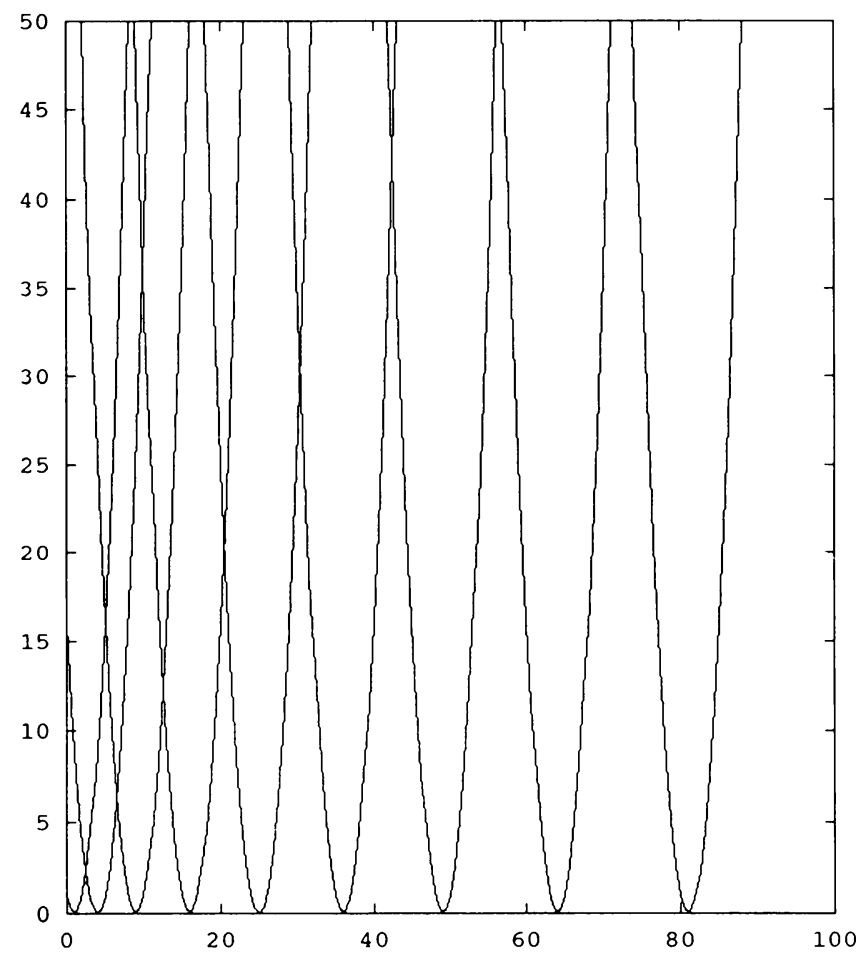

FIG. 2. Graph of $y^{\prime}(0)=\left(\lambda-p^{2}\right)^{2}$ for $p=1,2,3,4,5,6,7,8,9$. 
Conclusions. Bifurcation can occur from a continuum of eigenvalues. The bifurcated branches are tangent to the trivial solution at each point of the continuum. The decay of the solutions is different along each branch and is the same decay of the eigenfunction of the linearized problem at bifurcation. This leads us to expect a similar behavior for the solutions of the full problem; namely, the existence of a continuum of branches labeled by their node of decay which bifurcate tangentially from the trivial solution.

In the example of bifurcation from the continuous spectrum the behavior that we found is very different from the one described for local equations. In our case branches are labelled by the frequency of oscillation at the point of tangency. In other studies branches with a different number of nodes (frequencies) coalesce at a point.

Regarding finite-dimensional approximations the two examples show a very different behavior. For the first problem a finite truncation $a_{n}=0$ for $n \geq N$ has always a nonsingular linearization and the only solution is the trivial solution.

In the second example the discretization wil! have an increasing number of eigenvalues and the tangent bifurcation will be resolved as the coalescence of two transverse bifurcations.

These two examples indicate that analytical results are necessary in order to interpret the behavior of the numerics. In the first case the wrong answer is obtained by the discretization while in the second case care must be exercised in the interpretation.

\section{REFERENCES}

[1] V. Benci and D. Fortunato, Does bifurcation from the essential spectrum occur?, Comm. Partial Differential Equations 6, 249-272 (1981)

[2] B. Chen, M. C. Jorge, and A. A. Minzoni, Bifurcation of solutions for an inverse problem in potential theory, Stud. Appl. Math. 86, 31-51 (1992)

[3] C. A. Stuart, Bifurcation from the essential spectrum, Lecture Notes in Math., vol. 1017, Springer, Berlin, 1983, pp. 575-596

[4] C. A. Stuart, Bifurcation from the continuous spectrum in $L^{p}(\mathbb{R})$, Internat. Ser. Numerical Math., vol. 79, Birkhäuser-Verlag, Basel, 1987, pp. 307-318

[5] C. A. Stuart, Self trapping of an electromagnetic field and bifurcation from the essential spectrum, Arch. Rational Mech. Anal. 113, 65-96 (1991)

[6] J. Toland, Global bifurcation for Neumann problems without eigenvalues, J. Differential Equations 44, 82-110 (1982) 